\title{
High-precision piezo-ejection ocular microdosing: Phase II study on local and systemic effects of topical phenylephrine
}

\author{
Tsontcho lanchulev*,1, Robert Weinreb², James C Tsai' ${ }^{1}$, Shan Lin $^{3}$ \& Louis R Pasquale ${ }^{4,5}$ \\ ${ }^{1}$ New York Eye \& Ear Infirmary of Mount Sinai, New York, NY, USA \\ ${ }^{2}$ Hamilton Glaucoma Center \& Shiley Eye Institute, UCSD, San Diego, CA, USA \\ ${ }^{3}$ UCSF Medical Center, San Francisco, CA, USA \\ ${ }^{4}$ Glaucoma Service, Massachusetts Eye \& Ear, Boston, MA, USA \\ ${ }^{5}$ Channing Division of Network Medicine, Brigham \& Women's Hospital, Boston, MA, USA \\ * Author for correspondence: Tel.: +617 216 4369; tianchul@yahoo.com
}

\begin{abstract}
Aim: Conventional eyedropper-delivered volumes (25-50 $\mu \mathrm{l})$ exceed the eye's usual tear-film volume (7 $\mu l)$ and precorneal reservoir capacity, risking overflow and ocular/systemic complications. Piezoelectric high-precision microdosing may circumvent these limitations. Results \& methodology: In this masked, nonrandomized, cross-over study, subjects $(n=12)$ underwent pupil dilation with topical phenylephrine (PE) administered by $32-\mu$ l eyedropper ( $2.5 \%$ or $10 \%$ formulation) and $8-\mu /$ electronic microdosing ( $10 \%$ formulation). Microdosing with PE-10\% achieved comparable peak dilation as $10 \%$ eyedropper-delivery and superior dilation to $2.5 \%$ eyedropper-delivery $(p=0.009)$ at $75 \mathrm{~min}$. Microdosing significantly reduced 20-min plasma PE levels versus PE10\% eyedropper; neither treatment altered heart rate/blood pressure. Eye irritation occurred significantly less frequently with microdosing than PE10\% eyedrops. Conclusion: Piezo-ejection PE microdosing achieves comparable biological effect as eyedropper dosing; reduced systemic absorption may decrease risk of systemic side effects.
\end{abstract}

First draft submitted: 15 September 2017; Accepted for publication: 16 October 2017; Published online: 27 October 2017

Keywords:adverse events $\bullet$ microdosing $\bullet$ ocular drug delivery $\bullet$ ocular medication $\bullet$ phenylephrine $\bullet$ piezo-ejection system • pupil dilation $\bullet$ systemic absorption

A major impediment to topical medication absorption into the anterior eye is the limited penetration of many drugs through the multilayered avascular corneal barrier [1,2]. Additional physical defenses that reduce ocular drug bioavailability include reflexive tearing and blinking, and nasolacrimal drainage, which together increase precorneal drug dilution and clearance from the eye [3,4]. Conventional eyedroppers deliver 25-50 $\mu$ l doses of ocular medications, and these volumes greatly exceed the reservoir and absorptive capacities of the eye [2,3,6]. Excess medication volume that overflows onto the periocular skin may cause dermatologic reactions due to the drug or to preservatives. Increasing delivery volume increases nasolacrimal drainage from the eye [7]. Superflous ocular topical medication that drains into the nasolacrimal system may be systemically absorbed through the mucosa and can potentially initiate adverse events (AEs), sometimes serious [8]. Drugs entering the body via the nasolacrimal apparatus by-pass hepatic first-pass metabolism similar to intravenous administration, thereby increasing immediate systemic bioavailability [8].

Reducing the volume of topical ocular medications avoids potential problems associated with drug overflow, and may increase local absorption by reducing lacrimal drug dilution and tear loss due to blinking and drainage [9]. Enhanced ocular uptake may also reduce the portion of drug otherwise available for systemic absorption, and the attendant risk of undesired side effects.

We previously reported the development of a precision piezo-ejection microdroplet delivery system for administering small volumes of topical eye medications [10]. This handheld electronic system delivered single-digit microliter volumes of an aqueous pupil dilating drug combination [phenylephrine hydrochloride (PE) and tropicamide]. Microdosing resulted in equivalent pupil dilation as substantially larger doses delivered by the conventional 
high-volume eyedropper route. In the clinic, PE is commonly used as a topical 2.5 or $10 \%$ aqueous solution to achieve pupil dilation for diagnostic and interventional ophthalmic procedures; additionally, PE is sometimes administered orally or as a nasal spray for its decongestant action [11]. All drugs, including PE, carry risk of AEs. The current cross-over study compared PE ocular microdosing to conventional eyedropper dosing of PE at two concentrations, and evaluated not only local ocular effects, but also assessed systemic absorption.

\section{Methods}

\section{Subjects, screening \& enrollment}

We recruited healthy subjects aged $\geq 18$ years old without any significant ocular pathology or underlying medical condition, which, based on the investigator's medical judgment, could pose a risk to the subject or confound the study results. Female subjects of child-bearing age provided a negative urine pregnancy test before receiving any study medications, and again at the study conclusion. Subjects felt to have a potentially occludable angle based on gonioscopic findings were excluded from enrollment. The study protocol was reviewed and approved by the Institutional Review Board at Oculos Clinical Research LLC, which oversaw performance of the study at the Sall Research Medical Center, Artesia, CA, USA during October and November of 2016. Study performance complied with the tenets of the Declaration of Helsinki and the Health Insurance Portability and Accountability Act. All subjects provided written informed consent.

At the screening visit, disease and medication histories were taken, and subject eyes were examined grossly and by slit-lamp. After screening and enrollment, subjects began the three-arm crossover study. The three study medications were delivered in the following sequence, 7 days apart (variation was allowed so long as at least 2 days separated visits): PE-2.5\% ophthalmic eyedrops (Paragon BioTeck Inc., OR, USA); PE-10\% ophthalmic eyedrops (Paragon); and 10\% PE ophthalmic solution packaged for microdroplet spray delivery (PE- $\mu \mathrm{D})$ from Eyenovia Inc., FL, USA. Thus, the study comprised a screening visit at approximately Day -7 , and treatment visits at Days 0,7 and 14. Randomization of delivery sequence was not attempted because clinical staff could not be masked to the use of obviously different delivery systems. The physician that performed measurements was unaware of the treatment drug. All study drugs, including EYN-1601, contained $0.01 \%$ benzalkonium chloride as a preservative.

\section{Study medication administration}

All medications were administered to both eyes by a trained technician as either two conventional 32- $\mu$ l eyedrops or two $8-\mu 1$ microdroplet sprays, delivered $5 \mathrm{~min}$ apart. Study timing $(\mathrm{t}=0)$ began at the time the first drop or microdroplet spray was administered. For microdroplet delivery, the piezo-ejection device was held $3-5 \mathrm{~cm}$ in front of the eye, the subject fixated on the device LED targeting light, and the activation button was pushed to emit a horizontal microdroplet stream to an upright subject head, as previously described [6]. The handheld microdosing device has an exchangeable lower section containing an ampoule reservoir prefilled with sterile ocular medication, which snaps into an upper ejection system containing piezo-electronics and batteries. For reference drug delivery, eyedropper bottles were inverted vertically over a rearward-tilted subject head.

\section{Outcome parameters}

The primary efficacy outcome was change in pupil diameter from baseline ( pupil size). Pupils were measured using a NeurOptics VIP-300 digital pupillometer (NeurOptics Inc., CA, USA) with an accuracy of $\pm 0.03 \mathrm{~mm}$. Pupil diameter was measured immediately before dosing and then 15, 30, 45, 60, 75, 120 and 180 min afterward. All pupil measurements were performed in the same exam room under consistent scotoptic lighting conditions.

Pharmacologic assessment included plasma PE concentration in venous blood samples drawn 20 min after each study drug was administered. Samples were collected in $\mathrm{K}_{3}$ EDTA anticoagulant-containing tubes and centrifuged to separate plasma from formed elements. Plasma aliquots were frozen at $-70^{\circ} \mathrm{C}$ and shipped on dry ice by overnight courier to PPC Laboratories (WI, USA) for PE analysis. Plasma samples were analyzed for unconjugated (free/bioactive) PE by liquid chromatography-mass spectrometry using a validated assay (P898) with a detection range of $10-2500 \mathrm{pg} / \mathrm{ml}$.

Safety assessments included blood pressure and heart rate measurement at 10, 15, 30, 45 and 60-min time points, and the occurrence of AEs at any time between enrollment and study conclusion. 


\begin{tabular}{|c|c|c|}
\hline Parameter & & Value $(n=12)$ \\
\hline Age, years, mean \pm SD & & $23.4 \pm 3.3$ \\
\hline Gender, female, n (\%) & & $5(41.7 \%)$ \\
\hline - Females of childbearing age & & $5(41.7 \%)$ \\
\hline - Females with negative pregnancy test & & $5(41.7 \%)$ \\
\hline \multirow{3}{*}{ Race, n (\%) } & Black & $0(0.0 \%)$ \\
\hline & White & $10(83.3 \%)$ \\
\hline & Other & $0(0.0 \%)$ \\
\hline \multirow[t]{2}{*}{ Iris color ${ }^{\dagger}, \mathrm{n}(\%)$} & Blue & $0(0.0 \%)$ \\
\hline & Brown & $10(83.3 \%)$ \\
\hline \multirow{3}{*}{ Ongoing ocular issues ${ }^{\ddagger}, \mathrm{n}(\%)$} & Dry eye syndrome & $2(16.7 \%)$ \\
\hline & Pinguecula & $1(8.3 \%)$ \\
\hline & Superficial punctate keratopathy & $2(16.7 \%)$ \\
\hline \multirow[t]{3}{*}{ Other medical issues, $\mathrm{n}(\%)$} & Asthma & $1(8.3 \%)$ \\
\hline & Infection, local, skin & $1(8.3 \%)$ \\
\hline & Vitamin D deficiency & $1(8.3 \%)$ \\
\hline
\end{tabular}

\section{Statistical analysis}

Comparisons of pupil dilation between PE- $\mu \mathrm{D}$ and each eyedrop group used repeated measures mixed analysis of covariance, with treatment and eye within subjects as repeated measures. Dunnett-Hsu adjustment was applied because PE- $\mu \mathrm{D}$ was compared with both PE-2.5\% eyedrops and PE-10\% eyedrops. We tested the hypothesis that PE- $\mu \mathrm{D}$ was noninferior to PE-2.5\% eyedrops and PE-10\% eyedrops in increasing pupil dilation. Plasma $\mathrm{PE}$ concentration, heart rate and blood pressure were compared among groups using a two-tailed $t$-test; all three plasma PE datasets were assessed using Grubb's test to detect outliers, and a single outlier per group was excluded where identified. p-values $<0.05$ were considered significant. Our statistical approach was deemed acceptable by the US FDA in exchanges regarding our $510(\mathrm{k})$ clearance and investigational new drug application status and plans. Analyses software included Excel (Microsoft Corp., WA, USA) and Prism v. 5.0 (GraphPad Inc., CA, USA). Data are presented as mean $\pm \mathrm{SD}$ or Standard error of the mean, or as $\mathrm{n}(\%)$, as indicated.

\section{Results}

\section{Subject selection \& demographics}

A total of 16 subjects were screened to identify 12 subjects who were qualified for receipt of the study medication. Out of of 16 individuals screened, one subject met all criteria at the screening visit, but displayed hypertension during the first Treatment visit, which was identified before receiving any study medication. This subject was thus deemed ineligible for continued study participation. Three additional individuals satisfied study criteria at the screening visit, but did not proceed to additional visits because the 12 subjects necessary for treatment had been achieved. The remaining 12 subjects met all study criteria, received all study medication, and were included in analyses. No subject was discontinued from study medication due to medical emergency, development of serious AEs, use of prohibited medication, or pregnancy.

Mean cohort age was $23 \pm 3$ years, with $42 \%$ female and $83 \%$ Caucasian (Table 1). Most participants (83\%) had brown irides. Two subjects had comorbid bilateral dry eye syndrome, one of whom reported using artificial tears as needed.

Mean baseline pupil diameter $(\mathrm{n}=24$ eyes) was similarly $5.92 \pm 0.75 \mathrm{~mm}, 6.08 \pm 0.66 \mathrm{~mm}$ and $6.0 \pm 0.63 \mathrm{~mm}$ on the first, second and third treatment day, respectively $(\mathrm{p}>0.05)$. All study eyes responded to topical mydriatic 
(A)



(B)

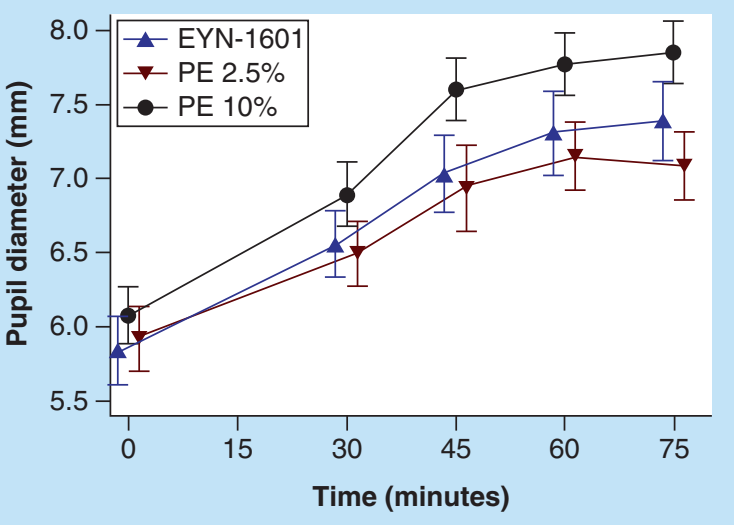

Figure 1. Pharmacodynamic pupil dilation. (A) Pupil diameter increase from baseline, at 30, 45, 60, and 75 min postadministration of PE-2.5, PE-10\% and PE- $\mu \mathrm{D}$, each respectively administered on study days 1,7 and 14 . (B) Average absolute pupil size closely paralleled the amount of pupil size change. Shown are mean \pm SEM for $n=24$ eyes of 12 subjects. Colored asterisk at $t=75$ min indicates significant difference versus comparator of asterisk color, in this case PE- $\mu \mathrm{D}$ versus PE-2.5\% $(p=0.009)$.

PE: Phenylephrine; SEM: Standard error of the mean.

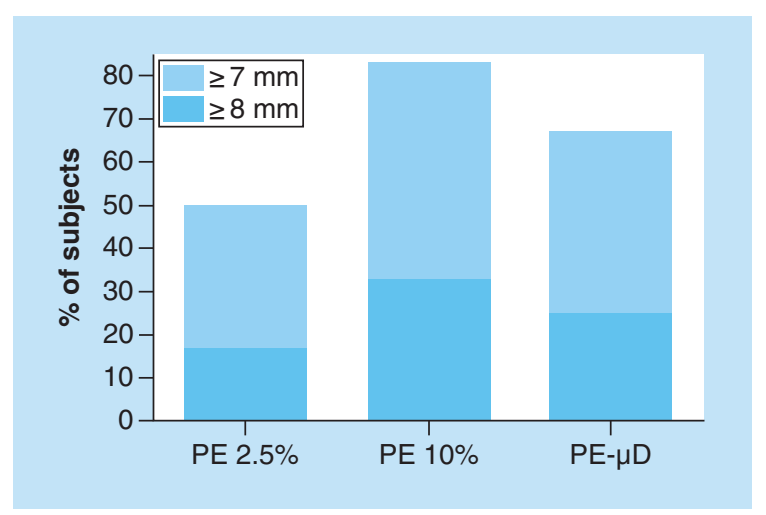

Figure 2. Range of pupil diameters $75 \mathrm{~min}$ after administration of phenylephrine-2.5, phenylephrine- $10 \%$ and phenylephrine- $\mu D$, each respectively administered on study days 1, 7 and 14 . This time point was prospectively selected to estimate maximal dilation effect. Shown are percentages of 12 average pupil diameters from 12 subjects (measurements of both eyes within each subject averaged before charting). Dilation to $\geq 8 \mathrm{~mm}$ was achieved in $25 \%$ of of $\mathrm{PE}-\mu \mathrm{D}$ microspray subjects, versus $17 \%$ of $\mathrm{PE}-2.5 \%$ conventional eye drop subjects and $33 \%$ of PE- $10 \%$ subjects. The proportion achieving $\geq 7 \mathrm{~mm}$ was $67 \%$ of PE- $\mu$ D subjects, $50 \%$ of PE- $2.5 \%$ eyedrop subjects and $83 \%$ of PE- $10 \%$ eyedrop subjects. PE: Phenylephrine.

agent application with a time-dependent increase in pupil dilation (Figure 1). PE- $\mu$ D resulted in a comparable $\Delta$ pupil size as PE-10\% eyedrops between 45 and $75 \mathrm{~min}$ (Figure 1A). At $\mathrm{t}=75 \mathrm{~min}, \Delta$ pupil size from baseline was similar in PE-10\% eyes $(1.78 \pm 0.73 \mathrm{~mm}$ dilation $)$ and PE- $\mu \mathrm{D}$ eyes $(1.55 \pm 0.71 \mathrm{~mm}$ dilation; $\mathrm{p}=0.318)$. Thus, the PE- $\mu \mathrm{D}$ microdroplet-induced pupil dilation was not inferior to that caused by PE- $10 \%$ delivered as conventional eyedrops. The difference in $\Delta$ pupil size between PE- $\mu \mathrm{D}$ microdose eyes and PE-2.5\% eyedrop (a full microdose of PE- $\mu \mathrm{D}$ ophthalmic solution $10 \%$ contains the same amount of the active ingredient as a standard PE-2.5\% drop) eyes grew increasingly larger between 45 and $75 \mathrm{~min}$ postadministration; this difference approached significance at $60 \mathrm{~min}(\mathrm{p}=0.084)$. Mean $\Delta$ pupil size in PE- $\mu \mathrm{D}$-treated eyes was $47.6 \%$ greater at $\mathrm{t}=75 \mathrm{~min}$ compared with eyes treated with PE-2.5\% (1.55 mm vs $1.05 \mathrm{~mm}$ size increase; $\mathrm{p}=0.009)$. The mean absolute pupil size plot closely paralleled the $\Delta$ pupil size plot (Figure 1B).

The distribution of pupil diameters 75 min after administration of PE-2.5, PE-10\% and PE- $\mu$ D is charted in Figure 2. Shown are percentages of 24 eyes from 12 subjects (measurements of both eyes within each subject averaged before plotting). The order of increasing percentages of treatment group eyes achieving pupil diameter $\geq 8 \mathrm{~mm}$ (from lowest to highest) was PE-2.5\% conventional eyedrops, PE- $\mu \mathrm{D}$ microspray, and PE-10\% conventional eyedrops. When using $\geq 7 \mathrm{~mm}$ as the cut off, at $75 \mathrm{~min}, 50 \%$ of PE- $2.5 \%$ conventional eyedrop subjects, $67 \%$ of PE- $\mu \mathrm{D}$ microspray subjects, and $83 \%$ of PE- $10 \%$ eyedrop subjects achieved this pupil diameter. 
Figure 3. Plasma phenylephrine concentration and distribution in venous blood that was drawn $20 \mathrm{~min}$ after ocular topical drug administration. Circulating free PE was highest in PE- $10 \%$ subjects $(316.3 \pm 36.8 \mathrm{pg} / \mathrm{ml})$, and was significantly $36.3 \%$ lower in PE- $\mu \mathrm{D}$ subjects (201.5 $\pm 27.1 \mathrm{pg} / \mathrm{ml} ; \mathrm{p}=0.021)$. Plasma PE was significantly lower in PE-2.5\% subjects (101.2 \pm $12.9 \mathrm{pg} / \mathrm{ml}$ ) than in PE- $\mu \mathrm{D}$ subjects $(p=0.003)$. Shown are means \pm SEM. $n=12$ subjects in the PE- $2.5 \%$ eyedrop group and 11 subjects in the PE- $10 \%$ eyedrop and PE- $\mu \mathrm{D}$ groups; a single outlier in each of the PE- $10 \%$ and $\mathrm{PE}-\mu \mathrm{D}$ groups was detected by Grubb's test and excluded.

PE: Phenylephrine; SEM: Standard error of the mean.

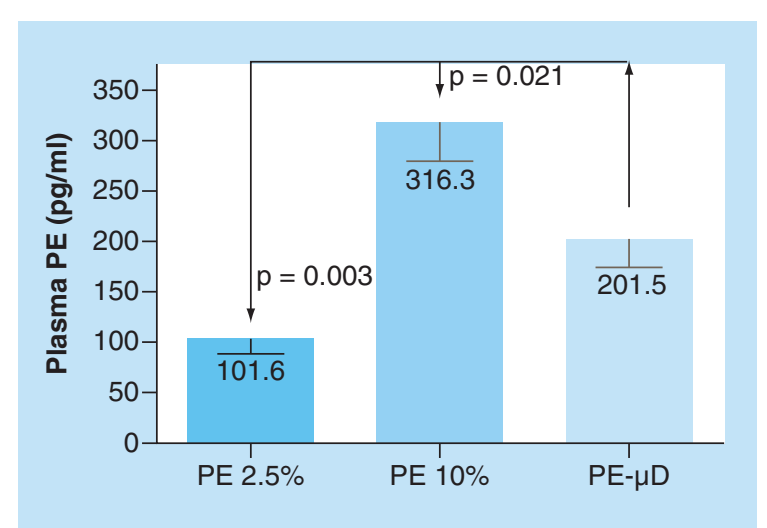

\section{Blood pressure \& pulse}

Average baseline systolic and diastolic blood pressures were similar after all three dosing regimens, and did not significantly differ from one another, or from baseline, at any follow-up time point through $60 \mathrm{~min}$ after drug administration (not shown). Average baseline heart rate in all three groups were also similar, and did not significantly differ from one another, or from baseline, at any follow-up timepoint through 60 min postdrug administration (not shown).

\section{Plasma PE level}

To quantify systemic absorption of ocular topical PE formulations, circulating plasma levels of free (bioactive) PE was measured in venous blood samples withdrawn from subjects $20 \mathrm{~min}$ after drug administration (Figure 3). The mean unconjugated PE levels in plasma from subjects that received PE-2.5, PE-10\% and PE- $\mu$ D measured 101.6, 360.8 and $237.8 \mathrm{pg} / \mathrm{ml}$, respectively. The circulating PE concentration in PE-microdosed subjects was lower than in PE-10\% controls that received a fourfold larger total PE dose (1.6 mg in PE- $\mu \mathrm{D}, 6.4 \mathrm{mg}$ in PE-10\% eye drops); this difference approached, but did not attain, significance $(\mathrm{p}=0.063)$. The plasma PE level in PE- $\mu \mathrm{D}$ subjects was 2.3 -fold higher than in PE-2.5\% controls $(\mathrm{p}=0.003)$, although both groups received the same total dosing of PE (1.6 mg).

\section{Subject comfort}

All subjects were queried about comfort at $45 \mathrm{~min}$ after administration of each of the three study drugs. Subjects were asked to rank comfort on a four-point scale of $0-3$, reflecting no, mild, moderate and severe discomfort. All 12 study subjects provided answers on all three drug administration days. On both the PE-2.5 and PE-10\% conventional eyedrop administration days, 1 of 12 subjects ( $8.3 \%$ ) reported 'mild discomfort', with the rest reporting 'no discomfort'. The subjects reporting mild discomfort with conventional eyedrops were different individuals. None of the 12 subjects reported any level of discomfort when queried 45 min after PE- $\mu \mathrm{D}$ administration.

\section{Adverse events}

All subjects underwent a slit-lamp examination at screening and again on all study drug administration days at $\mathrm{t}=0 \mathrm{~min}$, immediately before drug administration, and again at $\mathrm{t}=180 \mathrm{~min}$. Apart from the baseline observations provided in Table 1, no additional findings, and no AEs, were noted during any of the slit-lamp examinations performed during the study, in any subject.

Any AE, whether ocular or systemic, that was observed by the investigator or reported by the subject at any time during the treatment visits was recorded. The case report form specifically queried the investigator at 30 - and 180-min time points after drug administration about the appearance of any AEs. Ten AEs were reported in seven individuals, none of which were serious and all of which were considered to be 'mild' in nature; 9/10 AEs were ocular AEs suspected to be related to the study drug (Table 2), and the single nonocular AE was a mild case of upper respiratory tract infection deemed unrelated to the study drug. Out of the nine ocular AEs, 7 (78\%) occurred after PE-10\% conventional eyedropper dosing, and one each occurred after PE-2.5\% and PE- $\mu \mathrm{D}$ administration. Out of the nine ocular AEs, five were mild cases of ocular stinging/burning/irritiation, two were blurry vision, and 


\begin{tabular}{|c|c|c|c|c|}
\hline Adverse event description & Visit & Events, $\mathrm{n}$ & Severity & Related? \\
\hline \multirow[t]{2}{*}{ Ocular blurriness } & PE $2.5 \%$ & 1 & Mild & Yes \\
\hline & PE $10 \%$ & 1 & Mild & Yes \\
\hline \multirow{2}{*}{$\begin{array}{l}\text { Ocular } \\
\text { burning/stinging/irritation }\end{array}$} & PE $10 \%$ & 4 & Mild, (all) & Yes \\
\hline & $P E-\mu D$ & 1 & Mild & Yes \\
\hline Ocular dryness & PE $10 \%$ & 2 & Mild (both) & Yes \\
\hline $\begin{array}{l}\text { Respiratory tract infection, } \\
\text { upper }\end{array}$ & Screening & 1 & Mild & No \\
\hline
\end{tabular}

two were ocular dryness. All ocular AEs occurred immediately after medication administration, were bilateral, and every one resolved without intervention, without sequelae, and before completion of that particular study visit.

The single AE experienced on the PE- $\mu \mathrm{D}$ treatment date was a case of ocular burning immediately after medication administration. The single AE experienced in the PE-2.5\% eyedrop group was a case of blurry vision after drug administration. One of the two subjects with a history of dry eye syndrome reported AEs, consisting of a respiratory tract infection before any drug administration and ocular dryness after receiving PE- $10 \%$. No AE resulted in a subject being discontinued from the study, and all AEs resolved without sequelae.

\section{Discussion}

Eyedroppers deliver large dose volumes that exceed the reservoir capacity of the eye $[1,3,5]$. The $32-\mu l$ eyedropper delivery volume used in this experimental series represents the 25-50 $\mu \mathrm{l}$ range that is typical for contemporary aqueous eyedrops [5]. However, this dropper volume together with the usual $7-10-\mu l$ tear volume exceeds the 30- $\mu 1$ normal tear storage capacity of the eye (reduced to $10 \mu \mathrm{l}$ with blinking) [3,6,9]. Volume overload can result in both medication spillage onto the cheek and accelerated drainage through the nasolacrimal apparatus where it can be absorbed through the mucosa $[7,10]$. Eyedrops also act as minor irritants that initiate the tearing and blinking reflexes thereby diluting the administered medication $[4,8]$. Together with the multilayered corneal barrier, these impediments to topical medication retention and uptake at the anterior eye are considerable $[1,2,11,12]$.

Delivering ocular topical drugs in very small volumes may increase local bioavailability [9]. Microdosing avoids medication spillage from overloading the eye's maximum tear volume. Using small volumes may also decrease reflex tearing and blinking, thereby limiting drug dilution and washout. Previously, we demonstrated the practical feasibility of using a novel handheld piezo-ejection system to deliver precise single-digit microliter doses of topical ocular medications [13]. The current Phase II cross-over study provides additional evidence that ocular microdosing is a safe and effective approach for delivering finely-calibrated low-volume doses of medications to the eye. This study focused on the ocular delivery of a single mydriatic medication, PE, a drug that is used by ophthalmologists and optometrists worldwide [14]. Microdose delivery of aqueous PE to the eye resulted in equivalent-to-superior mydriasis compared with conventional eyedropper overdosing. An electronically-regulated 8- $\mu 1$ microdose of PE$\mu \mathrm{D} 10 \%$ showed comparable pupil-dilating action compared with high-volume conventional eyedropper PE-10\% administration, and superior dilation versus a drug-equivalent eyedropper dose of $2.5 \% \mathrm{PE}$. Thus, microdosing may increase medication bioavailability and uptake at the cornea.

Although, many approaches have been taken to increase ocular drug delivery [1,2,12], only a limited number of studies have explored microdosing aqueous ocular pharmacologics in humans. Previous experimental systems for low-volume ocular dosing were cumbersome devices that used modified syringes or air-injection cannulas, and were unsuitable for subject self-administration and home use. One group experimented with a microspray device based on a respiratory nebulizer but apparently discontinued those efforts without publishing any follow-on data regarding device reliability, dosing repeatability, or manufacturing costs [15]. In neonates, an 8- $\mu$ l droplet of PE-2.5\% produced the same dilation as a $30-\mu \mathrm{l}$ drop administered to the contralateral eye [16]. In adults, equivalent pupil dilation occurred with $10-\mu \mathrm{l}$ versus $30-\mu \mathrm{l}$ topical doses of PE-2.5\% [17]. Another study in adults used similar dosing parameters as our current study, and reported that bilaterally administered 8 -ul doses of PE- $10 \%$ caused significantly greater dilation than the same total drug amount delivered as $32-\mu \mathrm{l}$ of a $2.5 \%$ solution [18]. Likewise, in the current study, microdosing with $8-\mu 1 \mathrm{PE}-10 \%$ caused significantly greater pupil dilation than the same drug amount delivered in a $32-\mu$ l eyedrop of PE-2.5\%, and equivalent dilation as an eyedrop of PE-10\% containing four-times the medication. Thus, microdosing markedly reduced the total amount of drug needed to achieve the 
same biological effect. This could be especially useful if microdosing also reduces the quantity of more expensive or rare ocular therapeutics (e.g., biologics, recombinants, gene therapy vectors) required to obtain the same biological activity as macrodosing.

Only a small amount of topically applied ocular medication is absorbed by the cornea, necessitating delivery of high concentration when using an eyedrop. Besides, direct through the cornea, topical drugs are also absorbed by other ocular structures including the conjunctiva and sclera [19]. The conjunctiva has a greater surface area than the cornea and is between 2 and $30 \%$ more permeable depending upon the drug [20].

Approximately, $80 \%$ of ocular topical medication drains through the nasolacrimal apparatus, where it can be systemically absorbed through the mucosa [21], and blinking increases drainage [22]. Ocular drugs absorbed by the conjunctiva and the nasolacrimal mucosa mimic intravenous injection delivery insofar as they are not susceptible to first-pass hepatic metabolism. Additionally, ocular medication in swallowed nasolacrimal secretions is theoretically available for absorption in the GI tract. Only a small fraction of the applied medication is actually absorbed directly into the eye, while there remain multiple opportunities for systemic absorption [22].

Absorption of topical ocular drugs into the general circulation is associated with diverse AEs that are occasionally serious $[8,23,24]$, and PE is not exempted [25,26]. PE is similar to epinephrine/norepinephrine in chemical structure and also has cardiovascular activity. Unintended systemic absorption of ocular PE can change blood pressure and heart rate. A systematic review of relevant randomized controlled trials indicated that these changes are only associated with PE-10\% eyedrops, which transiently increase systolic blood pressure by $15 \mathrm{mmHg}$, and not the $2.5 \%$ concentration [26]. Although, these changes typically resolve within $60 \mathrm{~min}$, even small alterations in blood pressure may have a negative impact on populations with predisposing comorbidities [24]. We detected no difference in blood pressure or pulse from baseline, after any treatment, though our study was not specifically powered for this purpose.

Smaller ocular PE drop size significantly reduces systemic absorption; in infants, reducing PE-2.5\% eyedrop size from 30 to $8 \mu \mathrm{l}$ decreased plasma PE by $>50 \%$ [16]. In the current study, systemic PE levels were lower after ocular microdosing versus eyedropper delivery of PE-10\%. Circulating PE levels after microdosing were nearly twofold elevated versus the identical drug amount given as PE-2.5\% eyedrops, suggesting increased overall bioavailability with microdosing. Our study could not determine where systemic uptake occurred. In this study, there was no measurable change in blood pressure or heart rate through 60 min after any PE treatment, which additionally supports the general safety of ocular PE microdosing, although our sample size was small.

Systemic uptake of other topical ophthalmic medications might deserve more attention than PE [8,23]. Topical $\beta$-adrenergic antagonists are frequently prescribed to reduce intraocular pressure in glaucoma, and these drugs carry risk of serious cardiopulmonary AEs [27]. Timolol effectively treats glaucoma, but is rapidly absorbed from the eye, with peak plasma levels occurring only $15 \mathrm{~min}$ after dosing, and bioavailability equivalent to $78 \%$ of the same intravenous dose [28]. Ophthalmic timolol can cause symptomatic bradycardia, cardiac conduction disorders, orthostatic hypotension, and syncope and falls, particularly in geriatrics [29], and impairs pulmonary function in asthmatics [30]. Similarly, reducing the size of clonidine eyedrops (from 70 to $15 \mu \mathrm{l}$ ) in glaucomatous volunteers prevented the hypotensive effect that occurred with the larger drops [31]. Because these AEs are dose-dependent, microdosing of these and other ocular medications might reduce the likelihood of developing systemic side effects. Microdosing might also improve the effectiveness of ocular medical treatment by limiting washout, thereby maintaining local drug concentrations on the surface of the eye.

Ocular AEs identified in this study were mild and temporary. Microdosing PE-10\% resulted in fewer ocular AEs ( $1 / 12$ subjects) than larger volumes of PE- $10 \%$ delivered by dropper ( $7 / 12$ subjects), and an equivalent number as PE-2.5\% eyedrops. This correlates well with earlier observations that subjects experienced less pain and tearing, reduced overflow and more overall satisfaction with the head-upright piezo-ejection microdosing device versus eyedropper delivery [13]. Thus, microdosing may provide the additional benefit of improved comfort versus eyedropper dosing.

Eyedropper dosing can be imprecise due to irregular or unsuccessful self-administration. In glaucoma patients observed while self-administering a single eyedrop, only $9 \%$ did so correctly [32]. An average of $1.8 \pm 1.2$ drops were dispensed, $31 \%$ of subjects missed their eye and hit the eyelid/cheek, and $76 \%$ of subjects made ocular/periocular contact with the dropper bottle tip. In another study, 17\% of glaucoma patients reported physical difficulty with eyedrop self-administration and relied on others for drop instillation [33]. In another report, physical difficulty with eyedrop instillation was one of the most common barriers to success in the $27 \%$ of glaucoma subjects who self-reported poor medication adherence [34]. We speculate that a microdosing system with better accuracy and 
convenience than eyedropper delivery might improve ocular self-administration consistency, and adherence to medication dosing schedules [35].

This study demonstrated different bioactivity and pharmacodynamics of ocular drugs that are delivered by microdosing versus conventional eyedropper overdosing. We provide additional support that microdosing PE is a safe and effective alternative to dropper administration, and believe that this observation is probably relevant to other ophthalmic drugs. Though our data quantified total systemic absorption of PE, they provide little insight into the relative involvement of different anatomical locales in drug uptake. It will be useful to compare corneal uptake kinetics and resulting anterior chamber concentrations of topical ocular drugs delivered by piezo-ejection microdosing versus high-volume dropper. It is also necessary to identify whether the incidence and severity of both ocular and systemic AEs correlates with plasma levels of ophthalmic medications. We acknowledge that the inability to double-blind this study regarding treatment sequence may have introduced undetermined bias into our evaluation, but see little way around this limitation because of the noticeably differing delivery systems. Nonetheless, because the observer who measured pupil diameters, the study's primary efficacy outcome, was blinded to treatment, we believe an acceptable level of bias control was achieved and the objectivity of pupil measurements was preserved. Also, systemic PE levels were objectively determined by an off-site laboratory that was completely blinded to sample history. Thus, we believe that we have achieved our primary study goals.

\section{Conclusion}

Piezo-ejection microdose delivery of topical PE achieves comparable biological effect as dropper dosing four-times the drug amount and dose volume, with less systemic absorption and no detectable impact on cardiovascular function.

\section{Future perspective}

Eyenovia's microtherapeutic approach is widely applicable in the treatment of many anterior segment diseases such as glaucoma, dry eye, allergic eye disease and many infectious and immunologic etiologies. Topical side effects such as conjunctival hyperemia, discomfort, irritation and prostaglandin-associated periorbitopathy are dose-related and may be significantly reduced with high-precision targeted microdosing. The electronic piezo-delivery platform opens immense possibilities for eHealth and patient monitoring, compliance tracking and communication and customized treatment approaches. Ocular microdosing of very small volumes could be useful in delivering other ocular therapeutics, including antibiotics, analgesics and potentially rare or expensive biologics. Piezo-ejection technology might also be combined with other emerging and evolving ocular therapeutic technologies such as nanoparticles, corneal/limbal stem cells, lysophilates, and gene therapy vectors.

\section{Financial \& competing interests disclosure}

T lanchulev, R Weinreb and J Tsai are consultants for Eyenovia Incorporated (FL, USA), the device manufacturer. T lanchulev is named on the device patent application. J Tsai is a consultant for Aerie Pharmaceuticals (CA, USA), Inotek Pharmaceuticals (MA, USA), and Shire plc (Dublin, Ireland). L Pasquale is a speaker for Bausch \& Lomb (NY, USA), and has received travel support from the Glaucoma Foundation-New York and research funding from the National Institutes of Health for work unrelated to the current study. S Lin is a consultant for Aerie Pharmaceuticals, Allergan plc, (Dublin, Ireland), Iridex Corporation (CA, USA), and Aleyegn Incorporated (CA, USA), and has received travel support and honoraria from Eyenovia. The authors have no other relevant affiliations or financial involvement with any organization or entity with a financial interest in or financial conflict with the subject matter or materials discussed in the manuscript apart from those disclosed.

The authors thank M Silverman (Biomedical Publishing Solutions, FL, USA) for scientific writing and statistical analysis services. Silverman's fee was paid by Eyenovia.

\section{Ethical conduct}

The study protocol was reviewed and approved by the Institutional Review Board at Oculos Clinical Research LLC, which oversaw performance of the study at the Sall Research Medical Center, Artesia, CA, during October and November of 2016. Study performance complied with the tenets of the Declaration of Helsinki and the Health Insurance Portability and Accountability Act. All subjects provided written informed consent. 
Summary points

Conventional eyedroppers deliver oversized doses that exceed the eye's absorptive \& storage capacities

- Trigger reflex blinking and tearing:

- Drug loss to spillage and increased drainage;

- Medication dilution from tearing.

- Overflow can irritate periocular tissues.

- Nasolacrimal mucosal absorption can cause systemic side effects:

- No hepatic first-pass metabolism;

- Cardiopulmonary and neurological risks with common topical ocular medications;

- Children and elderly are at particularly high risk of systemic adverse events.

Piezoelectric delivery can precisely deliver single-microliter microdoses

- Overflow, ocular irritation and reflex blinking/tearing reduced:

- Less drug loss and medication dilution;

- Increased bioavailability to eye;

- Reduced local drug reactions;

- User-friendliness may increase compliance with ocular dosing regimens.

- Systemic drug absorption and related side effect risk are decreased.

Microdosing as effective as standard eyedroppers that deliver much larger doses

- Pupil dilation with phenylephrine similar between microdosing and eyedropper overdosing.

- Piezoelectric microdosing technology may be modified to deliver glaucoma and dry eye medications, antibiotics, analgesics, biologics, gene therapy vectors, nanoparticles, and corneal/limbal stem cells.

- Handheld electronic platform will allow individually tailored dosing regimens, and permit automated patient reminders and compliance monitoring, if desired.

\section{Open access}

This work is licensed under the Attribution-NonCommercial-NoDerivatives 4.0 Unported License. To view a copy of this license, visit http://creativecommons.org/licenses/by-nc-nd/4.0/

\section{References}

Papers of special note have been highlighted as: $\bullet$ of interest; $\bullet \bullet$ of considerable interest

1 Morrison PW, Khutoryanskiy VV. Advances in ophthalmic drug delivery. Ther. Deliv. 5(12), 1297-1315 (2014).

- A well-written review of evolving strategies to increase ocular drug bioavailability.

2 Washington N, Washington C, Wilson CG. Ocular drug delivery. In: Physiological Pharmaceutics: Barriers to Drug Absorption (2nd Edition). CRC Press, FL, USA, 249-270 (2001).

3 Mishima S, Gasset A, Klyce SD Jr, Baum JL. Determination of tear volume and tear flow. Invest. Ophthalmol. 5(3), 264-276 (1966).

4 Rucker JC. Normal and abnormal lid function. Handb. Clin. Neurol. 102, 403-424 (2011).

5 Van Santvliet L, Ludwig A. Determinants of eye drop size. Surv. Ophthalmol. 49(2), 197-213 (2004).

6 Scherz W, Doane MG, Dohlman CH. Tear volume in normal eyes and keratoconjunctivitis sicca. Albrecht Von Graefes Arch. Klin. Exp. Ophthalmol. 192(2), 141-150 (1974).

7 Chrai SS, Makoid MC, Eriksen SP, Robinson JR. Drop size and initial dosing frequency problems of topically applied ophthalmic drugs. J. Pharm. Sci. 63(3), 333-338 (1974).

8 Izazola-Conde C, Zamora-de la Cruz D, Tenorio-Guajardo G. Ocular and systemic adverse effects of ophthalmic and non ophthalmic medications. Proc. West. Pharmacol. Soc. 54, 69-72 (2011).

-• Assesses studies published between 1971 and 2010 concerning: ocular side/adverse effects of ophthalmic drugs; ocular side/adverse effects of systemic drugs; and systemic side/adverse effects of ophthalmic drugs.

9 Martini LG, Embleton JK, Malcolmson RJ, Wilson CG. The use of small volume ocular sprays to improve the bioavailability of topically applied ophthalmic drugs. Eur. J. Pharm. Biopharm. 44(2), 121-126 (1997).

10 Maliborski A, Rózycki R. Diagnostic imaging of the nasolacrimal drainage system. Part I. Radiological anatomy of lacrimal pathways. Physiology of tear secretion and tear outflow. Med. Sci. Monit. 20, 628-638 (2014).

11 Novack GD, Robin AL. Ocular pharmacology. J. Clin. Pharmacol. 56(5), 517-527 (2016).

- Current review of ocular anatomical and physiological barriers to topical ocular medication bioavailability, and morbidity associated with systemic absorption of topical ophthalmic drugs.

12 Patel A, Cholkar K, Agrahari V, Mitra AK. Ocular drug delivery systems: an overview. World J. Pharmacol. 2(2), 47-64 (2013). 
13 Ianchulev T, Chayet A, Kahook M, Packer M, Pasquale L, Weinreb RN. Pharmacodynamic profile of mydriatic agents delivered by ocular piezo-ejection microdosing compared with conventional eyedropper. Ther. Deliv. 7(11), 751-760 (2016).

-. The index report on the development of a new piezo-ejection ocular microdosing system and initial investigation into the safety and efficacy of using it to deliver topical pupil dilating medications.

14 National Library of Medicine; PubChem open chemistry database. PubChem CID 5284443. Phenylephrine hydrochloride. https://pubchem.ncbi.nlm.nih.gov/compound/phenylephrine_hydrochloride\#section=Human-Toxicity-Excerpts

15 Diestelhorst M, Hellmich M, Hochrainer D, Roessler G, Steinfeld A, Zierenberg B. The microspray: a new ocular drug delivery device. Invest. Ophthalmol. Vis. Sci. 46(13), 2469 (2005).

16 Lynch MG, Brown RH, Goode SM, Schoenwald RD, Chien DS. Reduction of phenylephrine drop size in infants achieves equal dilation with decreased systemic absorption. Arch. Ophthalmol. 105(10), 1364-1365 (1987).

- An earlier report that demonstrated equivalent pupil dilation but significantly reduced circulating plasma phenylephrine levels in neonates that received $2.5 \%$ topical ocular phenylephrine in an $8-\mu l$ volume $(0.9 \mathrm{ng} / \mathrm{ml})$ versus a $30-\mu \mathrm{l}$ volume $(1.9 \mathrm{ng} / \mathrm{ml})$.

17 Whitson JT, Love R, Brown RH, Lynch MG, Schoenwald RD. The effect of reduced eyedrop size and eyelid closure on the therapeutic index of phenylephrine. Am. J. Ophthalmol. 115(3), 357-359 (1993).

18 Brown RH, Wood TS, Lynch MG, Schoenwald RD, Chien DS, Jennings LW. Improving the therapeutic index of topical phenylephrine by reducing drop volume. Ophthalmology 94(7), 847-850 (1987).

-. A similar study design and primary outcome as the current report, in which significantly greater pupil dilation occurred with an 8- $\mu 1$ microdose of phenylephrine $10 \%$ versus a $32-\mu 1$ eyedrop dose of phenylephrine $2.5 \%$ containing the same total drug amount.

19 Hosoya K, Lee VH, Kim KJ. Roles of the conjunctiva in ocular drug delivery: a review of conjunctival transport mechanisms and their regulation. Eur. J. Pharm. Biopharm. 60(2), 227-240 (2005).

20 Ahmed I, Gokhale RD, Shah MV et al. Physicochemical determinants of drug diffusion across the conjunctiva, sclera and cornea. $J$. Pharm. Sci. 76, 583-586 (1987).

21 Maurice DM, Mishima S. Ocular pharmacokinetics. In: Pharmacology of the eye. Handbook of experimental pharmacology. Sears ML (Ed.). NY, USA, Springer-Verlag, 6919 (1984).

22 Urtti A, Salminen L. Minimizing systemic absorption of topically administered ophthalmic drugs. Surv. Ophthalmol. 37(6), 435-456 (1993).

23 Farkouh A, Frigo P, Czejka M. Systemic side effects of eye drops: a pharmacokinetic perspective. Clin. Ophthalmol. 10, 2433-2441 (2016).

24 Diamond JP. Systemic adverse effects of topical ophthalmic agents. Implications for older patients. Drugs Aging 11(5), 352-360 (1997).

25 Fraunfelder FW, Fraunfelder FT, Jensvold B. Adverse systemic effects from pledgets of topical ocular phenylephrine 10\%. Am. J. Ophthalmol. 134(4), 624-625 (2002).

26 Stavert B, McGuinness MB, Harper CA, Guymer RH, Finger RP. Cardiovascular adverse effects of phenylephrine eyedrops: a systematic review and meta-analysis. JAMA Ophthalmol. 133(6), 647-652 (2015).

-• A comprehensive overview of randomized controlled trials published between 1970 and $2014(\mathrm{~N}=916$ subjects in eight trials) indicating that $10 \%$ phenylephrine applied topically to the eye rapidly but transiently increases blood pressure and heart rate.

27 Vander Zanden JA, Valuck RJ, Bunch CL, Perlman JI, Anderson C, Wortman GI. Systemic adverse effects of ophthalmic beta-blockers. Ann. Pharmacother. 35(12), 1633-1637 (2001).

28 Korte JM, Kaila T, Saari KM. Systemic bioavailability and cardiopulmonary effects of $0.5 \%$ timolol eyedrops. Graefes Arch. Clin. Exp. Ophthalmol. 240(6), 430-435 (2002).

29 Mäenpää J, Pelkonen O. Cardiac safety of ophthalmic timolol. Expert Opin. Drug Saf. 15(11), 1549-1561 (2016).

30 Morales DR, Dreischulte T, Lipworth BJ, Donnan PT, Jackson C, Guthrie B. Respiratory effect of beta-blocker eye drops in asthma: population-based study and meta-analysis of clinical trials. Br. J. Clin. Pharmacol. 82(3), 814-822 (2016).

31 Petursson G, Cole R, Hanna C. Treatment of glaucoma using minidrops of clonidine. Arch. Ophthalmol. 102(8), 1180-1181 (1984).

32 Gupta R, Patil B, Shah BM, Bali SJ, Mishra SK, Dada T. Evaluating eye drop instillation technique in glaucoma patients. J. Glaucoma 21(3), 189-192 (2012).

33 Tony Tsai, Alan L. Robin, Judson P. Smith III. An evaluation of how glaucoma patients use topical medications: a pilot study Trans. Am. Ophthalmol. Soc. 105, 29-35 (2007).

34 Newman-Casey PA, Robin AL, Blachley T et al. The most common barriers to glaucoma medication adherence: a cross-sectional survey. Ophthalmology. 122(7), 1308-1316 (2015).

35 Waterman H, Evans JR, Gray TA, Henson D, Harper R. Interventions for improving adherence to ocular hypotensive therapy. Cochrane Database Syst. Rev. 4, CD006132 (2013). 
-• A systematic review of 16 randomized controlled trials $(n=1565)$ published through June 2012 that evaluated interventions to improve adherence to sometimes complicated ocular topical medication regimens. The authors highlight the lack of physician consensus in adopting approaches to overcome the challenge of long-term self-medicating regimens. 
